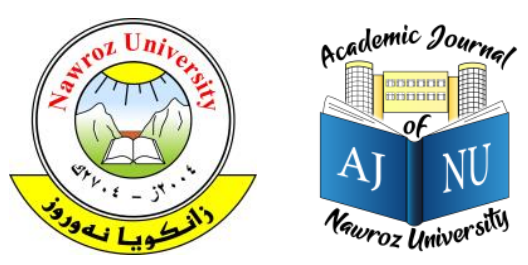

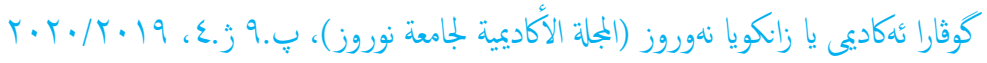

حقوق الطبع والنشر (2017. هذه مقالة الوصول اليها مفتوح موزعة تحت رخصة

e-ISSN: 2520-789X ،CC BY-NC-ND 4. 0. - المشاع الايداعي النسبي

https://doi.org/10.25007/ajnu.v9n4a991

\title{
المسؤولية عن الأضرار الناتجة عن استخدام الطائرات \\ بدون طيار " الدرون " في القوانين الداخلية و القانون الدولي
}

أ.م. د. شاري خالد معروف، قسم القانون، جامعة سوران جامعة نوج، اقليم كوردستان- العراق لقاق

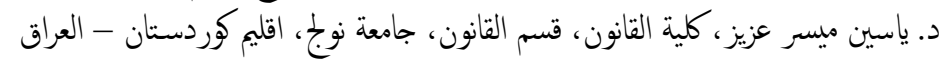

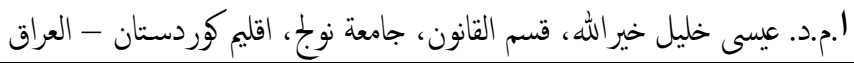

ان موضوع جثنا هو ( المسؤولية عن الاضرار الناتجة عن استخدام الطائرات بدون طيار "الدرون" في القوانين الداخلية والقانون الدولي) وقد درسنا فيه القوانين الداخلية فيا يتعلق بالمسؤولية عن استخدام هذا النوع من الطائرات التي بدون طيار ،وقد اوجدنا فقاط التقاء المسؤولية الدولية عن استخدام هذه الطائرات في النزاعات المسلحة مع الاتفاقيات الدولية المنظمة لقواعد الحرب من خلال مقدمة في البحث عن تاريخ الطيران واههيته وخمسة مطالب، تناولنا في المطلب الاول ماهية الطائرة بدون طيار ، والثاني استخدامات الطائرة بدون طيار "الدرون" ، والثالث المسؤولية القانونية عن استخدام الطائرات بدون طيار في القانون الدولي ، والرابع المسؤولية القانونية عن استخدام الطائرات بدون طيار في القوانين الداخلية ، والخامس عن شروط الاعفاء من المسؤولية عن الاضرار الناتجة عن استخدام الطيارات بدون طيار، وفي الخائة اوردنا اهم الاستنتاجات والتوصيات ذات الصالة بالبحث ومنها سن نصوص قانونية خاصة في القوانين الداخلية تحدد ضوابط استخدام الطائرات المسيرة والأار القانونية النترتبة على استخداما وعدم ترك الموضوع للاجتهادات والقياس، ووضع اتفاقية دولية في اطار الام المتحدة تنظم استخدام الطائرات المسيرة في حالات الحروب والنزاعات المسلحة. الكلمات المفتاحية: الطائرات بدون طيار - القواين الداخلية - القانون الدولي - الاتفاقيات الدولية المنظمة لقواعد الحرب.

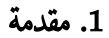

يحاول هذا البحث الموجز الإجابة على هذه التساؤلات وتحديد الأساس التي تبنى عليه المسؤولية عن استخدام هذه الطائرات ضمن القوانين الداخلية والدولية، كما ويحاول البحث ايجاد نقاط التقاء المسؤولية الدولية عن استخدام هذه الطائرات في النزاعات المسلحة مع الاتفاقات الدولية المنظمة لقواعد الحرب. لذا فقد ربط معظم ذوي الاختصاص القانون الجوي بالطائرات، فأصبح هذا القانون هو الذي يحكي حركة الطائرات وما ينشأ عنها من وقائع وعلاقات(1). وقد عرفت الطائرة بأنها مركبة أثقل من الهواء تعمل بقوة محركة وتستمد قوة رفعها من ردود فعل حركة الهواء على أسطح تبقى ثابتة في ظروف معينة. كا تم تعريف الطائرة بأهها "أي الة في استطاعتها ان تسيتمد بقاءهـا في الجـو مـن ردود فعل الهواء وليس بسبب ردود فعل الهواء المنعكسـة مـن سـطح الارض، وتثـمل كافـة المركبـات الهوائيـة، مثـل المناطيـد والبالونات والطائرات الشراعية والطائرات ذات الاجنحة الثابتة وما الى ذلك"(2). والطائرات من الأموال المنقولة وفقاً لما استقر عليه الفقه والقضاء وهو مـا تم النص
أثثار ظهور طائفة مـن تكنولوجيات الطائرات الجديدة مؤخراً عدداً مـن الأسئلة القانونية بما في ذلك أسئلة تتعلق بالقانون الدولي لحقوق الإنسان مـ جهـة، والقوانين الداخلية من بحة اخرى فيا يتعلق بالمسؤولية عن استخدام هذا النوع الجديد من الطائرات. حيث أن تكنولوجيا الطائرات بلا طيار تطورت تطوراً ملحوظاً منذ ظهورهـا قبـل 15 عامـاً، وأن اسـتخدام هـذه الطائرات زاد زيادةً هـائلة في سـياق النشاطات المدنية والعمليات العسكرية وتدابير مكافة الإرهاب. ويسعى عدد متزايد من الدول إلى الحصول على تكنولوجيا الطائرات بلا طيار، وعند الوقوف أمام العدد الهائل المتصاعد من هذه الطائرات يوما بعد آخر ، ثمة أسـئلة منطقية تفرض نفسها عن ماهيـة هـذه التكنولوجيا التي أدخلت العالم في مضـار تسـابق وتنـافس غير مسبوقين والتي تدفعنا إلى التساؤل عا إذا كانت الطائرات المسيرة تثثل تقنية من شأهها أن تخرج عن المجالات التقليدية للمسؤولية الدولية والداخلية؟ والى أي مـدى ستصل تأثيراتها المستقبلية في تغير الرؤية الإستراتيجية مدنياً وعسكرياً 


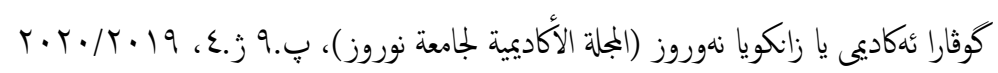

المسوؤلية الدولية عن الاضرار التي قد تلحق عند استخدام هذا النوع من الطائرات

\section{1}

اعتمد البحث على المنهج (الوصفي التحليلي) , فكما هو معلوم ان هذا المنهج يعتمد على دراسة الظاهرة كما توجد في الواقع وبهتم بوصفها وصفأ دقيقأ.وذلك بهدف توضيح مواطن الغموض التي تكشف المسوؤلية عن الاضرار الناتجة عن استخدام هـا النوع من الطائرات .

\section{1}

تم تقسيم هيكليـة البحثث المى خمس مطالب بالاضـافة المى مقدمة وخاتمة , وهي كالاتي:

- م المطلب الاول: ماهي الطائرة بدون طيار. - المطلب الثاني: استخدامات الطائرة بدون طيار "الدرون".

- المطلب الثالث: المسوؤلية القانونية عن استخدام الطائرات بدون طيار في

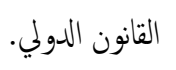

- المطلب الرابع : المسوؤلية القانونية عن استخدام الطائرات بدون طيار في

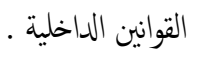

- المطلب الخـامس: شرط الوعفاء مـن المسـوؤلية عن الاضرار الناتجة عـن

$$
\text { استخدام الطائرات بدون طيار . مان }
$$

\section{2. - 2 - المطلب الأول: ماهية الطائرة بدون طيار.}

يطلق على الطائرة بدون طيار في اللغة الانجليزية اسم ( Unmanned Aerial (Vehicle اختصاراً للمصطلح (UAV)، وكتسمية شعبية في بعض البلدان العربية، ولما يتميز به صوت هذه الطائرة أثناء التحليق تسمى أيضاً بالزنانة، وهي طائرة توجه عن بعد أو تبرمج مسبقاً لطريق تسلكه، وفي الغالب تحمل حمولة لأداء مهامها، كأجهزة وكاميرات أو حتى القذائف وإن الاستختدام الأكبر لها هو في الأغراض العسكرية كالمراقبة والهجوم، لكن شهـ استخدامها في الأعمال المدنية في مكافحة الحرائق ومراقبة خطوط أنابيب النفط والغاز تزايداً كبيراً، حيث تستخدم في المهام الصعبة والخطرة بالنسـبة للطائرة التقليديـة والتي يجـب أن تـتزود بالعديـد مـن احتياجات الطيار مثل المقصورة ، وأدوات التحكم في الطائرة ، والمتطلبات البيئية،
عليه في عدة تشريعات عربية، ومنها قانون الطيران المدني رقٌ (148) لسـنة 1974 المعدل، وأجنبية ومنها ما نصت عليه المادة (6121) من قانون النقل الفرني- رقة (1307) لسـنة 2010(3). وقد نصـت المادة (40) مـن قانون الطيران المـدني رقة (148) لسنة 1974 المعدل، على إن "الطائرة مال منقول فيا يتعلق بتطبيق القوانين والانظمة النافذة في الدولة ومع ذلك فان نقل ملكية الطائرة يجب ان يتم بموجب سـند رسمي ولا يكون له اي اثر تجـاه الغير الا بعـد قيـده في السـل الخـاص بذلك"(4). وتكن الانسان بعد ذلك من إحداث تطويرات هائلة على النموذج الأولي للطائرة التي تم اختراعها، ووصلت إلى ما نحن عليه من تقنيات الطيران بدون طيار. وكان للأخوين الأمريكيين "رايت"(5) الفضل في اختراع الطائرات بدون طيار إلى حيز الوجود ولأول مرة في العالم عام 1903. وقد أثبتـت الطائرة في الحرب العالمية الثانية أهميتها العظمة في المساعدة الفعلية في حسم المعارك لصاح الجانب الأقوى (6). 1.1 اهمه البحث تتجلى هذه الاهمية في عدة نقاط كمة سواء على المستوى الداخلي (مستوى الدولة في نطاقها الجنرافي) او على مسستوى الدولي, ومن النقاط التي تبرز اهية هـا

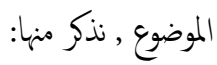
- مدى اهمية استخدام هذا النوع من الطائرات في الجوانب المدنية والعسكرية - تحديد المسوؤلية تجاه الاثـخاص والجهات التي تستخدم هـذا النوع من

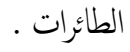
- تحديد التعويض في المسوؤلية المدنية من جهة وكيفية اثباتها من جهه اخرى .

2.1 ههدف البحث الى النعرف على الطائرات المسيرة والتعرف على خصائص ومجال استخدام هذا النوع من الطائرات في الجوانب الانسانية والعلمية ونقل الاثـاص والبضائع والحروب . 3.1 مشكلة البحث ان اشكلية هـا الموضوع تتمحور حول موضوع المسوؤلية , اي المسوؤلية عن الاضرار الناتجة عن استخدام هذا النوع من الطائرات , وبتالي الزام الجهة بالتعويض عن هذه الاضرار ولا سيها في ظل التعاون الدولي وذلك من اجل تطوير قواعد 


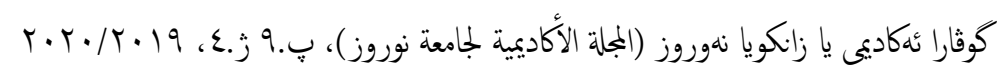
مثل الضغط والأوكسجين وأدى التخلص من كل هذه الاحتياجات في الطائرات

\section{3 نقل البضائع والسلع.}

قامت شركة أمازون الأمريكية بتطوير طائراتها لنقل وتوزيع البضائع المطلوبة من موقع الشركة، وكذلك قيام الطائرات بدون طيار العائدة لهذه الشركة بتوصيل السلع للعملاء، إذ أن هذه الطائرات مزودة بتكنولوجيا تهدف إلى الاستفادة من نظام تحديد المواقع المعروف بصطلح Global Positioning System أو GPS فتمنت من إيصال السلع والبضائع، للزبائن بدقة وفي غضون 30 دقيقة من طلب شرائها. أما شركة غوغل فإن طائراتها التي تستخدم في عمليات توصيل الطلبات تيتع

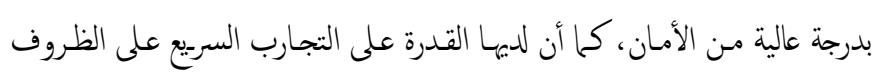
الطارئة مثل الرياح والأمطار وغير ذلك من الظروف الجوية(10).

\section{3 نقل الأشخاص}

ابتكرت شركة صينية، الطائرة الأولى مـن نوعها بـدون طيار تستطبع أن تنقل

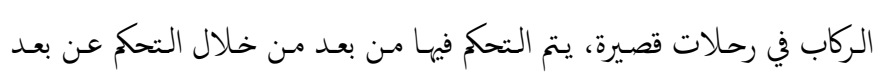

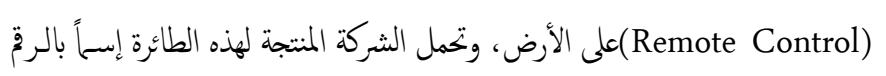
(184)، وهي عبارة عن تأكي طائرة، تعمل بالطاقة الكهربائية. كحا أعلنت الشركة

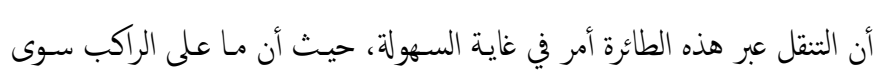
تحديد الوجحة التي يريد السفر إلها، ومن ثم يطلب عبر تطبيق هاتني الوجهة فيتم الأمر (11).

\section{3 التصوير في مجال السينا والأفلام الوثاثقية والأحداث الرياضية.}

تستخدم الطائرات بدون طيار في مجموعة أخرى مـن الأغراض كالتصوير السينائي وبجم الأخبار، وذلك للحصول على مسح شامل للحدث أو الحصول على معلومات لبث تقرير وثائتي تلفزوني أو إذاعي، وعادة ما يستخدمون في هذا النوع الكاميرات

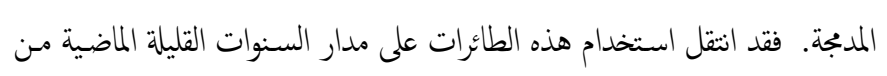
كونه حصراً على المصورين المحترفين إلى أمر في متناول الجميع، متيحاً بذلك لمبي لمبي التصوير إمكانية الحصول على أفضل النتائج (12). وبضضل قدرتها على حمل كاميرات

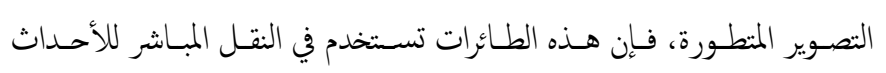
الرياضية، كباريات كرة القدم، وسباق السيارات، وتسلق المرتفعات الجبلية وغيرها

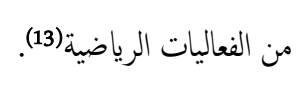

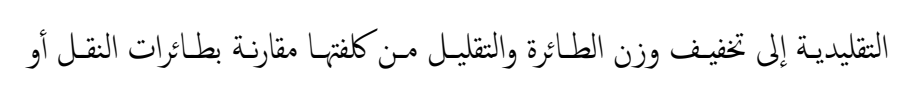
الطائرات الحربية. لقد غيرت هذه الطائرة من طبيعة الحرب الجوية، بجيث أصبح المتحكى في الطائرة غير معرض للخطر الحقيقي الذي يتعرض له الطيار بسبب العمليات العسكرية، أو الحوادث التي تواجه الطائرات المدنيـة بسبب سوء الأحوال الجوية، أو العطل لئل

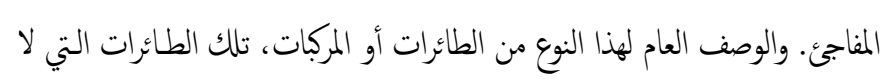
يوجد بها طيار، وتطلق إلى الجو بالطريقة الاعتيادية، أي تدحرج، وتقلع باستعمال

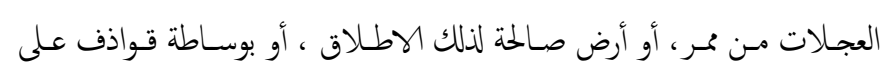

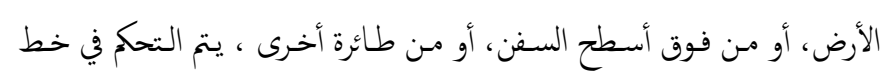
مسارها، وفي السيطرة على الأظظمة الفنية فيها لاسلكياً، عن بعد ؛ لتقوم بالطيران إلى منطقة عملها ؛ لأداء مهمتها والعودة إلى قاعدتها ؛ ليعاد استعحالها مرة أخرى. وهذه الطائرات تبرمج وتوجه عن بعد، ويتحكم فيها خبراء متخصصون على الأرض، وتكون مجهزة بأدوات تسمح لها بأداء المهام المطلوبة، وقد تكون مزودة بأبهزة

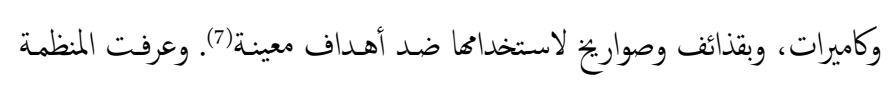
الدولية للطيران المدني هذا النوع من الطائرات بأنها طائرات بدون طيار على متهها(8). وتشغل الطائرات بدون طيار بمساعدة شخص يتحكم بها ويوجتها من على الأرض، كما يتم تزويد الطائرة قبل اقلاعها ببرنامج يحوي جميع أهـداف المهمة والخطة الموضوعة، سواء أكانت المهمة عسكرية أم مدنية. ويككن تحديث هذا البرنامج أثناء طيران الطائزة بتعديل المهمة أو إلغائها، كما إنه يكن لهذه الطائزة التحليق في الجو بلا مساعدة المتحكم بها في حالة فقدان الاتصال بها(9).

3. المطلب الثاني: استخدامات الطائرة بدون طيار" الدرون " بضل التطور الكبير في التكنولوجيا المستخدمة فيها، فإن استخدام الطائرات مـن دون طيار قد اتّسع ليشمل عدة مجالات منها النجاري، كخدمات توصيل المشتريات،

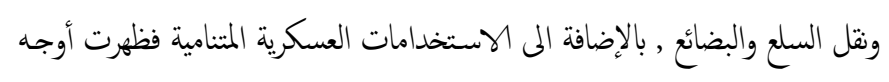
عديدة لاستخدام الطائرات بدون طيار في عمليات التوصيل كتوزيع البريد والوثائق الإدارية عوضا عن البريد التقليدي، كما تم استخدامها أيضا في العمليـات التجارية كنقل البضائع والسلع للمستهاكين. 


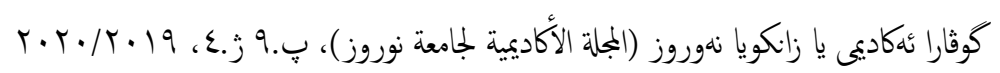

ضررها، يكون مسؤولاً عا تحدثه من ضرر، ما لم يثبت انه اتخذ الحيطة الكافية لمنع

وقوع هذا الضرر، هذا مع عدم الاخلال بما يرد في ذلك من احكام خاصة"(18).

4.3 المساعدات الطبية.

وهذا ما نصت عليه الفقرة الأولى مـ المادة (1384) من القانون المدني الفرنسي-،

المتعلقة بالمسؤولية الناشئة عن حراسة الأشياء غير الحية(19).

وقد انتسم الفقه والقضاء بشأن الأشياء التي تتطلب في حراستها عناية خاصة إلى اتجاهين، إذ يرى الاتجاه الأول أن الأشياء التي تتناج في حراستها إلى عناية خاصة أما أن تكون طبيعها تقتضي مثل هذه العناية، وإما أن لا نكون الأشياء خطرة في

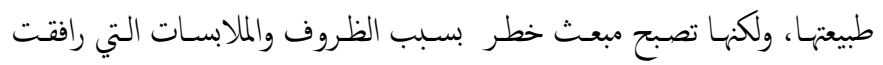
الحادث، لذلك فقد اقتضت عناية خاصة في حراستها ولو لم تكن خطرة في الأصل ، فالشجرة على سبيل المثال ليست شيئا خطرا إذا كانت في وضعها الطبيعي ، ولكنها تصبح خطرة إذا اقتلعتها الرياح ورمت بها على قارعة الطريق، أي انه بحسب هـذا الاتجاه تعتبر الأشياء بحاجة ماسة لعناية في حراستها إذا كانت خطرة بطبيعتها أو إذا كانت خطرة بملابساتها(20). أما الاتجاه الثاني فيرى أن الأشياء التي تحتاج في حراستها إلى عناية خاصة هي الأشياء الخطرة بطبيعتها ، أمـا الأشـياء التي لا يعتبر الخطر ملازم لطبيعتها فلا تتطلب عناية خاصة في حراستها ، ومن ثم لا تدخل في تطبيق المادة 231 مـدني عراقي والمادة 178مدني مصري، ويستندون إلى ما ورد في الأعمال التحضيرية للتقنين المدني المصري ، إذ تقول المذكرة الإيضاحية، أن المشروع لن يمضي- في هذا السبيل لأقصى من غايته ، بل اجتزء ما تقتضيه أحوال البلاد في المرحلة الراهنة مـ حياتها الاقتصادية(21).

وبالتالي فان هذه المسؤولية تتحقق بتوافر شرطين يتمثل الشربط الأول بـأن يتولى شخص حراسة شيء تقتضي- حراسته عناية خاصة، أي وجود هـا الشيء عند تسببه في حدوث الضرر في حراسة الشخص المراد مساءلته عن ذلك مسؤولية مفترضة. أما الشرط الثاني فهو أن يقع الضرر بفعل ذلك الشيء، ومن مقتضىـهذا الشرـط ألا يكون الضرر قد وقع بسبب فعل الإنسان مباشرة، بل بواسطة شيء (22). ويرى شراح القانون أن الأشياء التي تستوجب حراستها عناية خاصة، إما بسبب طبيعتها، وإما بسبب وضعها في ظروف معينة تضاعف احتمال حدوث ضرر منها للغير (23). 


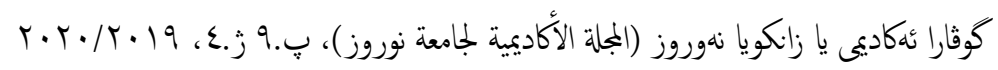

لم يرد ذكر الطائرات بدون طيار بشكل محدد في معاهـات الأسلحة أو صكوك القانون الدولي الإنساني الأخرى. ومع ذلك فإن استخدام أى نظام من نظم الأسلحة بما في ذلك الطائرات المسلحة بدون طيار أثناء النزاعات المسلحة يخضع بوضوح

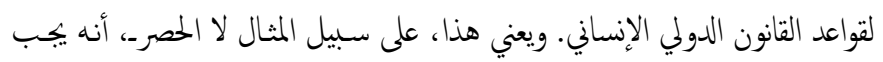
على أطراف نزاع ما عند استخدام الطائرات بدون طيار أن تميز دائماً بين المقاتلين والمدنيين وبين الأهداف العسكرية والأهداف المدنية. ويجب عليها اتخاذ جميع التدابير الاحترازية الممكنة لحقن دماء السكان المدنيين وحاية البنية التحتية المدنية ويجب لئب عليها تعليق الهجوم أو إلغؤه إذاكان الأذى أو الضرر العرضي المتوقع على المدنيين أو الأهداف المدنية مفرطاً مقارنة بالميزة العسكرية الملموسة والمباشرة المتوقعة. وبالمثل لا لا يجوز بأي حال استخدام الطائرات بدون طيار في نقل أسلحة مخظورة مثل المواد الكيميائيسة أو البيولوجيـة. ومـن ناحيـة أخـرى، وحسـب منظـور القـانون الدولي الإنساني، ينبني تفضيل استخدام أي سلاح يجعل من الممكن تنفيذ هجات دقيقة ويساعد، بصفة عرضية، على تجنب أو تقليل إحداث خسائر في أرواح المدنيين أو إلحاق الإصابة هم أو الإضرار بالأعيان المدنية، على استخدام الأسلحة التي لا تتسم بالدقة نفها ـ وتحدد الظروف المحددة ما إذاكان استخدام الطائرات المسلحة بدون طيار يحقق هذه المزايا بالفعل. وأصبح هذا الموضوع محل نقاش مسيتمر ، بسبب قلة المعلومات عن تداعيات معظم ضربات الطائرات بدون طيار ، على سبيل المثال لا الخصر. وعندما تُستخدَ الطائرات بدون طيار في حالات لا يوجد فهيا نزاع مسلح، ينطبق القانون الوطني ذو الصلة والقانون الدولي لحقوق الإنسان ومعايير إنفاذ القانون التي ينص عليها، وليس القنانون الدولي الإنساني. أن الكثير من الدول استخدمت نموذج الحرب استخداماً غير ملائم في تحديد سياق عمليات مكافة الإرهاب وأن ذلك ينطبق أيضاً على استخدام الطائرات المسلحة بلا

\section{طيار (29)} ان المتحكمين في أنظمة الأسلحة التي يجري تشغيلها عن بعد مثل الطائرات بدون طيار بعيدين عن ساحة القتال بيد أنهم يديرون نظام الأسلحة ويحددون الهدف ويطلقون الصواريخ. ويعمل المتحكمون في تشغيل الطائرات بدون طيار بشكل عام تحت قيادة مسؤولة، ومن ثم فهم وقيادتهم يكونون عرضة للمساءلة عا يحدث بموجب القانون الدولي الإنساني. إن وجود المتحكمين في تشغيل الطائرات بدون طيار عن
وقد اسـتقر الفته الفرنسيـ على إن المسؤولية الناشـئة عن حراسـة الأشـياء غير الحية(24)، تطبق على الطائرات بدون طيار، كون المستخدم لهذه الطائرات، هو من يعمل على سلطتي التوجيه والرقابة، وبالنتيجة يكون مسؤولاً عن الأضرار التي يتعرض لها الأفراد من جراء هذا الاستخدام(25.). وإن المسؤولية المتتبة عن الأضرار الناتجة عن استخدام الطائرات بدون طيار تقوم على أساس الخطأ المفترض من له سلطة توجيه هذه الطائرة افتراضاً لا يقبل إثبات العكس. ولا يستطيع الشخص المسؤول التخلص مـن هذه المسؤولية، إلا باثبـات السبب الأجنبي الذي لا يد له فيه، سواء أكان قوة قاهرة، أم خطأ مـن المضرور، أو خطأ الغير (26)، كأن يتم اعتراضها من طائرة أخرى، أو التشويش عليها من مصدر خارجي. علماً بأنه لا يكن التخلص من المسؤولية، إلا إذاكان القائم على الرقابة والتوجيه ملتزماً بشروط استخدام هذه الطائرة المحلية أو الدولية التي التزمت بها الدولة. وإن هــه الشرـوط لم تـنص عليها الاتفاقـات والبروتوكولات والمعاهــات الدوليـة، كاتفاقية وارسو عام 1929، واتفاق لاهـاي عام 1955، وبروتوكول غواتيهالا عام

ومن الشروط التي وضعتها الدول المعنية باستخدام الطائرات بدون طيار ، كالولايات المتحدة الأمريكية وروسيا واليابان ودولة الإمـارات العربية المتحدة والصين وكندا، حصول ترخيص الترخيص بالطيران صادر من الجهة الختصة، وعدم التجاوز على الحياة الخاصة للأفراد، وعدم تجاوز ارتفاع الطائرة عن 150 مـراً، على أن تشـاهد بالعين المجردة، وأن لا يقل عمر القائم على السيطرة والتوجيه عن سن معينة، وكذلك عدم التحليق بالقرب من المطارات بمسافة تقل عن 55؟(27).

5. المطلب الرابع: المسؤولية القانونية عن استخدام الطائرات بدون طيار في القانون الدولي(28). (28)

لا تحظر قواعد الحـرب المنصوص عليها في القـانون الدولي الإنساني، وهي جموعة القوانين التي تنظم النزاعات المسلحة، صراحة استخدام الطائرات بدون طيار، ولا تعتبرها عشوائية أو غادرة بطبيعها. وهي لا تختلف في هذا الصدد عن الأسلحة التي تطلقها طائرات يقودها طيار مثل المروحيات وغيرها من الطائرات المقانلة. ومع ذلك

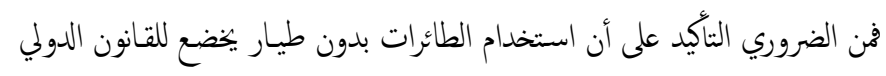
رغز أن الطائرات مشروعة في حد ذاتها. 


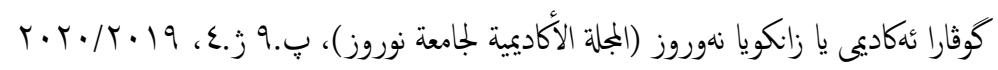

من المعلوم إن المسؤولية المدنية تقام عندما يخل الشخص بما التزم بـه تجاه الغير، سواء أكان إخلالاً بالتزام قانوني أم بإلتزام عقدي(36)، وإن الجزاء المتزتب عن هـا الإخلال هو تعويض الضرر الناثئ عن ذلك الإخلال، وذلك طبقاً لما نصت عليه المادة (202) من القانون المدني العراقي، والمادة (163) من القانون المدني المصري، والمادة (1382) من القانون المدني الفرني-. وإن المسؤولية التي تنشأ عن الأضرار التي تسبها الطائرات بدون طيار للطائرات الأخرى في الجو أو تلك الجاثمة في أرضية المطارات، أو للأهداف الحيوية والأموال العامة، أو عن الأضرار التي تصيب الأفراد على الأرض، قد تكون مسؤولية تقصيرية، إذ إن كلاً من المسؤول والمضرور يعد بكيم الأجنبي بالنسبة للآخر. وغالباً ما نرى مثل هذه الحالة عند استخدام الطائرات بـدون طيـار لأغراض التسلية، أو في العـروض التزفيهـة. وإن سبب قيـام هـذهـ المسؤولية، هو نتيجة عدم إتخاذ الحيطة والحذر في عمل المسؤول، مما تسبب بالضرر الذي كان بالإمكان تلافيه. ويستوجب لقيام هذه المسؤولية أياً كان نوعها، توافر كافة شروطها(37)، وتتمثل هذه الشروط في خطأ ارتكبه من له سلطة السيطرة والتوجيه الطائرة بدون طيار، وحصول ضرر بسبب هذا الخطأ، كأن يكون سقوط الطائرة على الأرض وإصـابة أحد الأفراد، أو حصول أضرار للمنشـآت العامة أو الخاصة، ووجود علاقة السبية بين الخطأ والضرر. وفي هذه الحالة، يكون شرط الإعفاء مـن المسؤولية الذي يتم الإتفاق عليه مسبقاً بين المضرور والمسؤول باطلاً لمخالفته النظام العام. أما لو كان الإتفاق قد حصل بعد وقوع الضرر، فلا نكون أمـام شرط الإعفاء

$$
\text { من المسؤولية، بل نكون أمام حالة صلح. }
$$
وقد تكون المسؤولية الناشـئة عن الأضرار التي تسبيها الطائرات بـدون طيار، ، مسؤولية عقدية، وذلك عندما توجد رابطة عقدية بين الطيار عن بعد أو من يتولى توجيه الطائرة، والشركة التي تمتلك هذه الطائرات لغرض التيام بنشاط زراعي، أو تقوم بنقل البضائع، أوإيصال الطرود البريدية أو القيام بعملية المسح الجيولوجي. وعليه فإنه إذا ما حصل أي خطأ أو تأخير في هذه الأعلال، وتسبب ذلك الخطأ بحصول أضرار وخسائر مادية للشركة، فننشأ مسؤولية من له سلطة الرقابة والتوجيه، ولا يمكن أن يتخلص منها إلا بإثبات السبب الأجنبي، كالقوة القاهرة، والحادث المفاجئ، وخطأ الغير. أما شرط الإتفاق على الإعفاء من هذه المسؤولية بين صاحب الرقابة والتوجيه من جهة والشركة المالكة من جهة أخرى، فهي صحيحة، ما لم يكن الأول قد

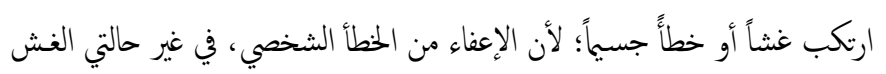

بعد من ساحة القنال لا يعفيهم هم ولا قيادتهم من مسؤولياتهم التي تشمل الالتزام بمبدأي التمييز والتناسب واتخاذ كافة التدابير الاحترازية اللازمة عند الهجوم. فلا فرق إذاً بين المتحكمين في تشغيل الطائرات بدون طيار وبين قائدي الطائرات مثل المروحيـات أو المقـاتلات الأخــى مـن حيـث الـتزامهم بالامتثـال للقـانون الدولي الإنساني، وبالتالي يجوز استهدافهم بموجب قواعد القانون الدولي الإنساني.(30) ويجب عند استخدام الطائرات المسلحة بلا طيار، الالتزام بالفقرة 4 من المادة 2 من ميثاق

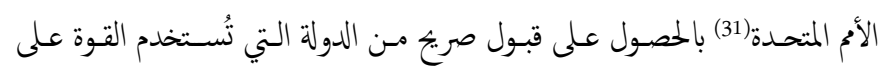

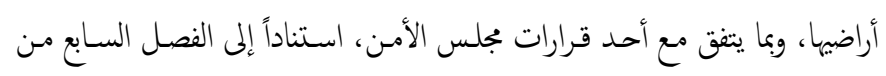
الميثاق، أو في الدفاع المشروع عن النفس في موابحة هوم مسلح، فإن استخدام القوة على أراضي دولة أخرى دون موافتهـا يـرق إلى عمل عدواني. حيـث يثير

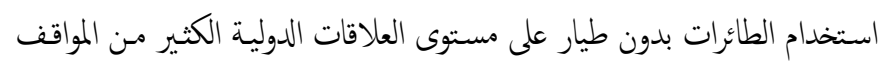
الـتي تـدعو الدولة المى المطالبـة بتطبيـق احسكام وقواعـد القـانون الدولي وضرورة احترامه(32) ، لذلك فقد اعتبر مقرر الام المتحة الخاص ب( حالات الاعدام خارج نطاق القضاء) بقوله " ان سياسة الادارة الامريكية باستخدام الطائرات بدون طيار لتنفيـذ قتل مستهـدف بهـدد اسس القانون الدولي " وذلك خلال مؤتمر عقد في جنيف منتصف عام 2012 (33) ، ولايخفى ان هناك ججود حثيثة من قبل منظطات الججتع المـني وحقوق الانسان نحو ادانة ( القتـل المستهدف) وذلك مـن خلال استخدام الطائرات بدون طيار بحجة مكافة الارهاب، فبالر غم من عدم وجود قواعد قانونية دولية حول استخدام هذه الانواع من الطائرات الا ان هناك احكام عامـة تفرضها قواعـ الاخلاق والانسـانية. وتجــر الاشـارة الى ان المـادة الثالثة المشتركة في اتفاقيات جنيف الاربع قد حرمت الاعمال اللاانسانية والتي يكننـا اعتبار استخدام الطائرات بدون طيار اثناء النزاعات المسلحة من ضنها(34) وخلاصة القول، فان الباحثي يقققون مع الرأي الذي يرى بان الدول التي ينتك مجالها

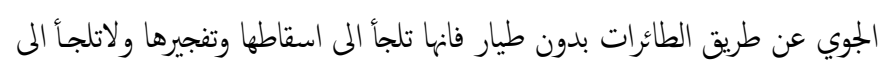
اثارة المسؤولية الدولة عن هذا الفعل، وربما يعود السبب في ذلك المى صعوبة اثبات الفالع خصوصا مع التطور الهائل لهذه الطائرات والتحكم بها عن مسافات بعيدة جدا وكل ذلك يؤدي الى صعوبة اخضاعها الى المراقبة الدولية(35) 6. المطلب الخامس: شرط الإعفاء من المسؤلية عن الأضرار الناتجة عن استخدام الطائرات بدون طيار. 


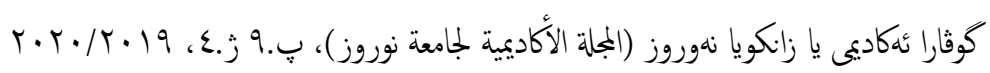

لا تحظر قواعد الحرب المنصوص عليها في القانون الدولي الإنساني صراحة استخدام الطائرات بدون طيار وأن الآثار المترتبة على استخدام الطائرات المسلحة بلا طيار وفق القانون الدولي تقع ضمن اختصاص مجلس حقوق الإنسان. يجب على أطراف نزاع ما عند استخدام الطائرات بدون طيار أن تميز دائماً بين المقانين والمدنيين وبين الأهداف العسكرية والأهداف المدنية. ويجب عليها. اتخاذ جميع التدابير الاحترازية الممكنة لحقن دماء السكان المدنيين وحاية البنية التحتية المدنية ويجب عليها تعليق الهجوم أو إلغاؤه إذاكان الأذى أو الضرر العرضي المتوقع على المدنيين أو الأهداف المدنية مفرطاً مقارنة بالميزة العسكرية

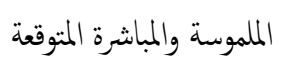

ان استخدام الطائرات المسيرة إذا أحدث ضرراً للغير نتيجة هذا الاستخدام؛ فإن هذا الضرر يرتب المسؤولية وفق قواعد حراسة الأشياء التي نصت عليها القوانين المدنية المختلفة

إن المسؤولية المتزتبة عن الأضرار الناتجة عن استخدام الطائرات بدون طيار تقوم على أساس الخطأ المفترض من له سلطة توجيه هذه الطائرة افتراضاً لا

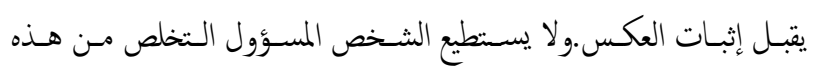
المسؤولية، إلا ياثثبات السبب الأجنبي الذي لا يد له فيه ان الإتفـاق عـلى الإعفـاء مـن مسؤولية الاضرار الناشـئة عـن اسـتخدام الطائرات المسيرة بين صاحب الرقابة والتوجيه مـن جهـة والشركة المالكة مـن

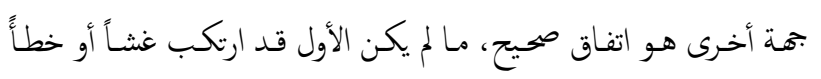

سـن نصوص قانونيـة خاصـة في القوانين اللاخليـة تحـد ضـوابط استخدام الطائرات المسيرة والأثار القانونية المتزتبة على استخداهما , وعدم ترك الموضوع للاجتهادات والقياس على القواعد العامة التي قد لا تكفي في كثير من الاحيان لمواجهة التطورات التكنوجية المتلاحقة في هذا المجال.
والخطأ الجسيم،جائز لدى غالبية الفقهاء(38). ويكن أن تكيف المسؤولية التي تسبها

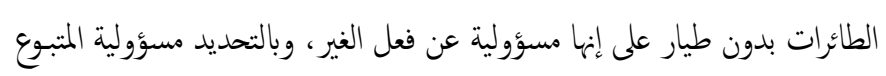
عن أعمال تابعه، إذ تغع هذه المسؤولية على عاتق الجهة المستفلة للطائزة، وتنشأ عندما يرتكب الطيار عن بعد أو من يتحكم بالتوجيه والسيطرة على الطائرة، خطاً يؤدي إلى حدوث ضرر يصيب الآخرين، وذلك وفقاً ملا جاءت به المادة (1/219) مـن القانون المدني العراقي التي تنص على إن "الحكومة والبلديات والمؤسسـات الاخرى التي تقوم بخدمة عامة وكل شخص يستغل احد المؤسسات الصناعية او التجارية مسؤولون عن الضرر الذي يحدثه مستخدموهم، اذاكان الضرر ناشسئً عن تعد وقع منهم اثناء قياهم بخدماتهم"(39). وفي ضوء ما تقدم آنفاً، فقد يتم الإتفاق بين مستغل الطائرة بدون طيار أو الجهة التي تعود لها الطائرة من ججة، والمضرور من جهة أخرى على إعفاء الطرف الأول من المسؤولية الناشئة عن فعل الغير ، طبقاً لنص المادة ( 2/259) مدني عراقي ، والمادة (2/217) من مدني مصري(40)، التي تقضي بجواز الاتفاق على اعفاء المدين من كل كل

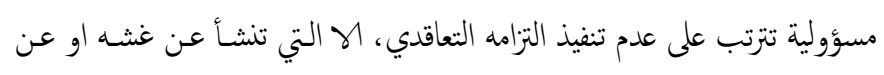
خطأه الجسيم، ومع ذلك يجوز المدين أن يشترط عدم مسؤوليته من الغش او الخطأ الجسيم الذي يقع من اشخاص يستخدمم في تنفيذ التزامه.

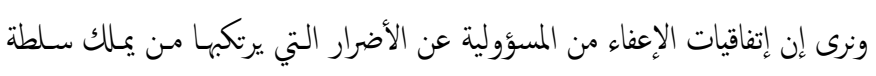
الرقابة والتوجيه للطائرة بدون طيار ليست كلها صحيحة، ويجب أن لا يؤخذ بها على لى لئل

إطلااقها. فعلى سبيل المثال ينبغي عدم التعامل بالجزء الأخير من النص، أي لايجوز للمدين،

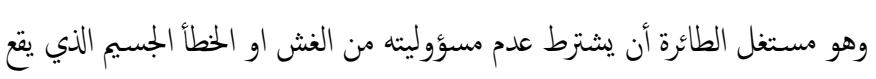
من اشخاص يستخدهم في تنفيذ التزامه. وبالتالي يكن إعفاء المدين عن الأخطاء البسيطة، كان يكون تأخيراً في توصيل البضاعة، أو عدم القيام بأعمال مسح ميداني لمنطقة ما نتيجة السبب الأجنبي، كالقوة القاهرة، أو خطأ الغير ، أو خطأ المضرور،

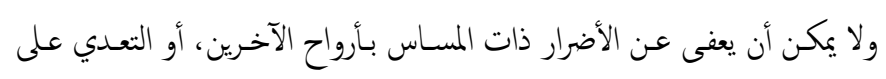
خصوصياتهم.

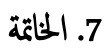
في خاتمة البحث نورد أهم الاستناجات والتوصيات ذات الصلة بالبحث وكما يلي: 1.7 الاستنتاجات 


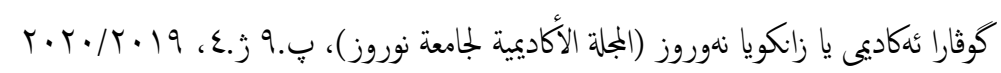

\section{8 المصادر الأجنية والانترنت}

1. Eminent Jurists Panel on Terrorism, Counter-Terrorism and Human Rights, Assessing Damage, Urging Action (International Commission of Jurists, Geneva, 2009).

2. Ordonnance n2010/1307 du 28 Octobre 2010, sur le site www.legifrance.gouv.fr

3. Simran, Brar and others. Drones for deliveries. Technical Report.Berkeley university. California. 2015.

4. Oween Bowcot, "Dron strikes thirteen 50 years of international. Says Un regporture" the Guardian 21/6/2012.

5. https://www.marefa.org/index.php-

6. https://www.mc-doualiya.com

7. https://www.ultraswat.com

8. https://ar.wikpedia.org/wiki

9. https://blogs.aljazeera.net/blogs

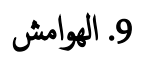

(1) محمد فريد العريني، القانون الجوي، دار المطبوعات الجامعية , 2012، ص5.

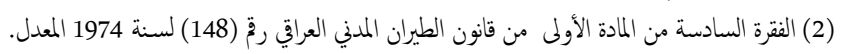

(3) Ordonnance n2010/1307 du 28 Octobre 2010, sur le $\quad$ site www.legifrance.gouv.fr

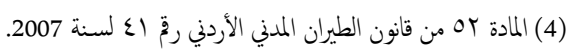

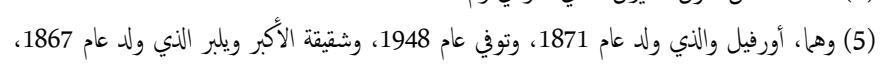
وتوفي عام 1912.

(6) طارق الراوي، الطائرات المسيرة، الطائرات بدون طيار، 2015، ص ص 20. tarekalrawi@yahoo.com (7) طاهر شوقي مؤمن، النظام القانوني للطائرات بدون طيار، كلية العلوم الإدارية والمالية، جامعة الملك

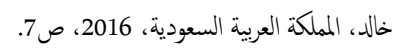

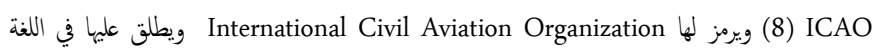
الانجليزية (9) طارق الراوي، الطائرات المسيرة، الطائرات بدون طيار، مرجع سابق، ص 18. (10) Simran, Brar and others.Drones for deliveries. Technical Report.Berkeley university. California. 2015. P06. On-line at: http://scet.berkeley.edu/wp-content/uploads/ConnCarProjectReport-1.pdf

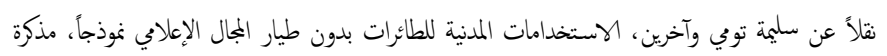

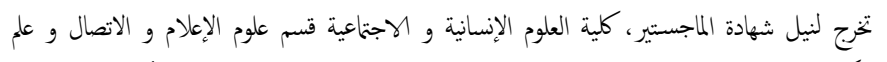

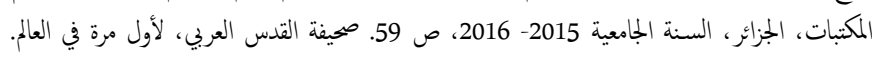

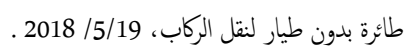
(11) صيفة القدس العربي، لأول مرة في العالم. طائرة بدون طيار لنقل الركاب، http://www.alquds.uk/?p=509427 (12) محمد العوضي، استعراض أحدث أنواع الطائرات بدون طيار، جريدة إيلاف الكاكترونية، 10 مارس 2017. http://elaph.com/Web/News/2017/3/1137454.html
وضع اتفاقية دولية في اطار الامح المتحدة تنظم استخدام الطائرات المسـيرة في

$$
\text { حالات الحروب والنزاعات المسلحة. }
$$

1.8

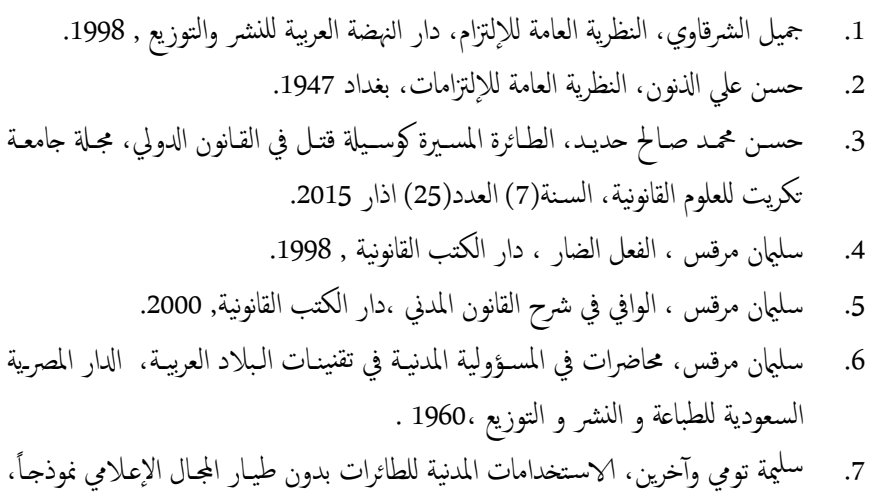

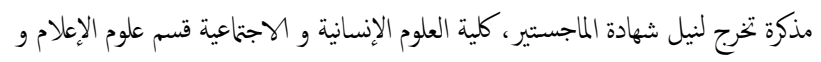

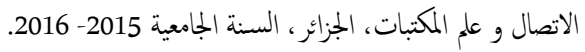

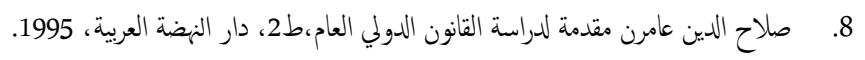

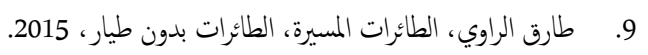

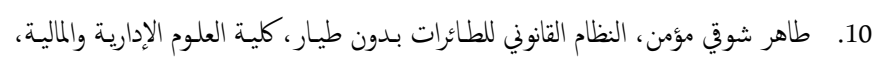

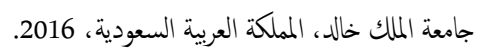

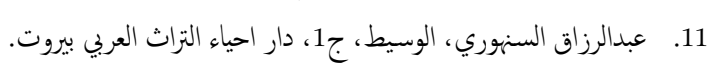

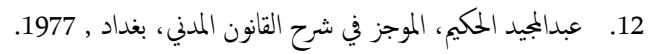
13. محمد فريد العريني، القانون الجوي، دار المطبوعات الجامعية , 2012.

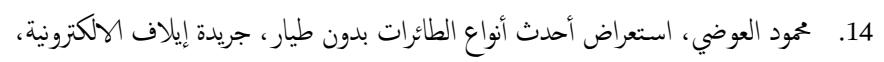
10 مارس 2017 . 20 15. محمود جلال حمزة ، العمل غير المشروع بأعتباره مصدر للالتزام ،ديوان المطبوعات ، الجزائر. 16. القوانين:

17. قانون الطيران المدني الأردني رقُ اعـ لسنة 2007. 18. قانون الطيران المدني العراقي رق (148) لسنة 1974 المعدل. 19. - الرسائل الجامعية:

20. بدر جاسم اليعقوب، المسؤولية عن الأشياء الخطرة، رسالة دكتوراه، حقوق القاهرة،

. 1977

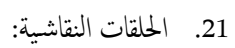

22. حلقة النقاش التفاعلية للخبراء التي عقدها مجلس حقوق الإنسان بشأن استخدام الطائرات

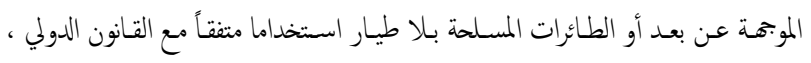

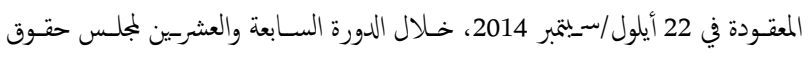

الإنسان. 


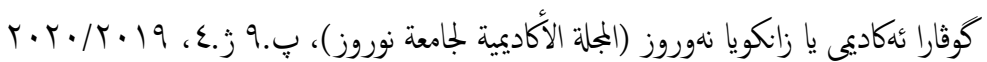

Owen Bowcotle, "Dron strikes thirteen 50 years of international.SaysUnrg ( 33) porture" the Guardian21/6/2012

(34 ) في حالة قيام نزاع مسلح ليس له طابع دولي في أراضي أحد الأطراف السامية المتعاقدة، يلتزم كل طرف في الزناع بأن يطبق كمد أدنى الأحكام التالية:

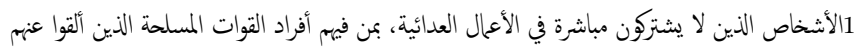

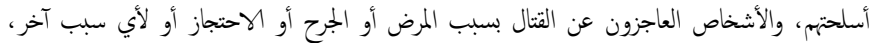

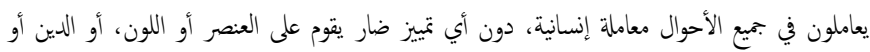

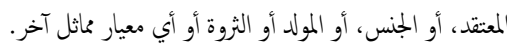
ولهذا الغرض، تخظر الأفقال التالية فيا يتعلق بالأشخاص المذكورين أعلاه، وتبقى مخظورة في جميع الأوقات آلمات والأماكن: (1) (أ) الاعتداء على الحياة والسلامة البدنة، وبخاصة القتل بجميع أشكاله، والتشويه، والمعامة القاسية،

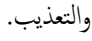

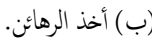

(ج) (ج) المتداء على الكرامة الشخصية، وعلى الأخص المعاملة المهينة والماطة بالكرامة.

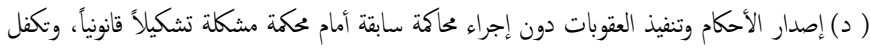
جميع الضمانات التضائية اللازمة في نظر الشعوب المتمدنة.

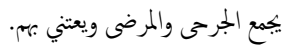

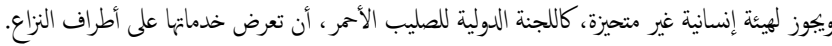
وعلى أطراف النزاع أن تعمل فوق ذلك، عن طريق اتفاقات خاصة، على تنغيذ كل الأحكام الأخرى من الني هذه الاثناقية أو بعضها. وليس في تطبيق الأحكام المتقدمة ما يؤثر على الوضع القانوني لأطراف النزاع. https://blogs.aljazeera.net/blogs. ( 35 )

(36) حسن علي الذنون، النظرية العامة للإلتزامات، بغداد 1947، ص 178.

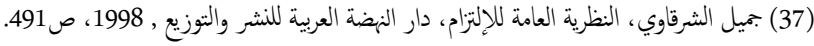

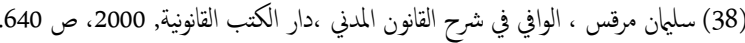

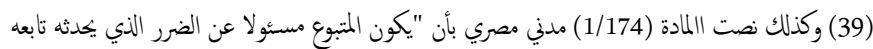

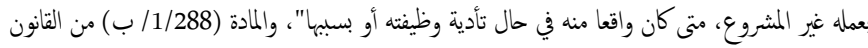

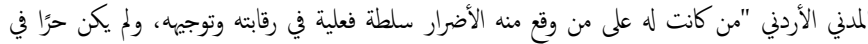
اختياره إذا كان الفعل الضار قد صدر من التابع في حال تأدية وظيفته أو بسبيها".

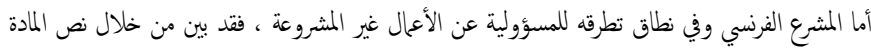

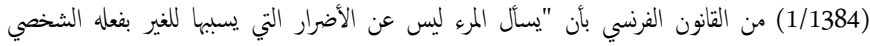

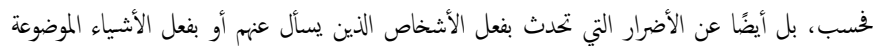

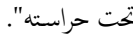

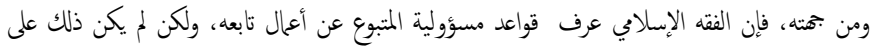

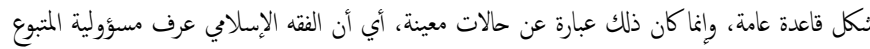

شكل محدود وضيق. (40) تنص المادة (2/217) من القانون المدني المصري "وكذلك يجوز الاتفاق على إعفاء المدين من أية

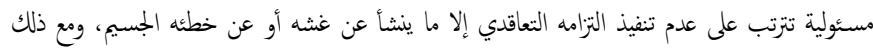

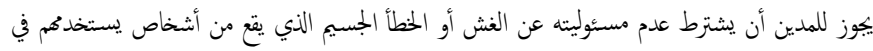
تنفيذ التزامه". http://www.makeuseof.com/tag/use-gopro-cameras-extreme-sports

نقلاً عن سليمة بن توي وآخرين، الاستخدامات المدنية للطائرات بدون طيار الججال الإعلاي نموذجاً،

مرجع سابق، ص84.

https://www.ultrasawt.com ( 14)

whttps://ar.wikpedia.org/wiki ( 15)

(16 ) في واقعة حديثة اصيب اربعة مدنيين في السعودية بحطام طائرة بدون طيار اطلقها الحوثيون باتجاه

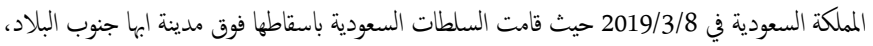
حسب ماافاد به التحالف الذي تقوده الرياض في اليمن. https://www.mc-doualiya.com

(17) عبدالجيد الحكيم، الموجز في شرح القانون المدني، بغداد , 1977، ص ص 585.

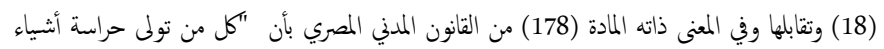

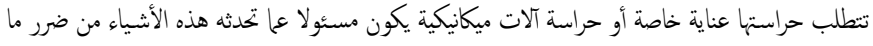

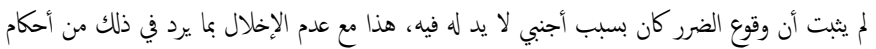

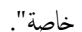
(19) محمود جلال حمزة ، العمل غير المثروع بأعتباره مصدر للالتزام ،ديوان المطبوعات ، الجزائر، ص202 صوا بعدها. (20) بدر جاسم اليعقوب، المسؤولية عن الأشياء الخطرة، رسالة دكتوراه، حقوق القاهرة، 1977 ، ص

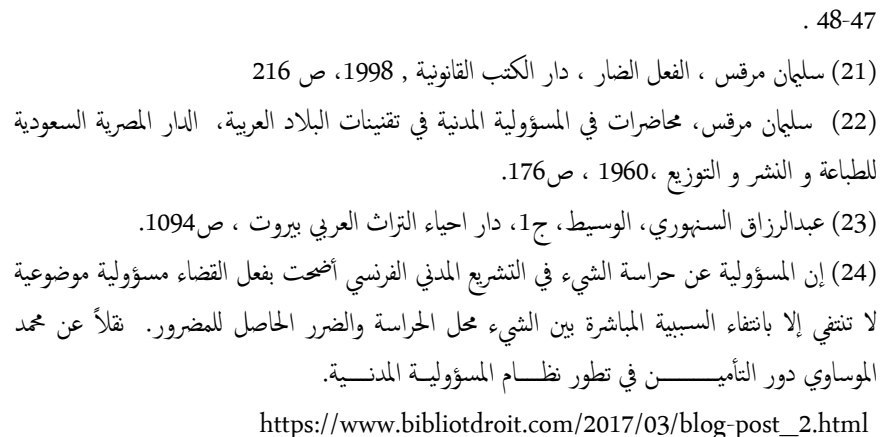
Selene, drones et aéronefs, une quasi - collision, le 10 Mars 2010, sur le (25) site, www.selene-avocats.fr/publications. نقلاً عن طاهر شوقي مؤمن، النظام القانوني للطائرات بدون طيار ،مصدر سبق ذكهه ، ص 29.

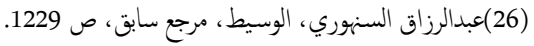

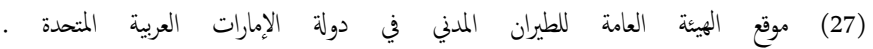
www.gcaa.gov.ae/ar/pages

(28 ) لمزيد من التفصيل ينظر حلقة النقاش النفاعلية للخبراء التي عقدها مجلس حقوق الإنسان بشأن

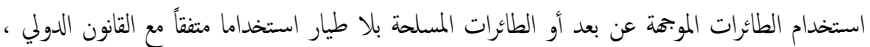
المعقودة في 22 أيلول/سبتمبر 2014، خلال الدورة السابعة والعشرين لمجلس حقوق الإنسان.

Eminent Jurists Panel on Terrorism, Counter-Terrorism and Human (29) Rights, Assessing Damage, Urging Action (International Commission of Jurists,

.Geneva, 2009) (30 ) د.حسن ممد صالح حديد، الطائرة المسيرة كوسيلة قتل في القانون الدولي، مجلة جامعة تكريت

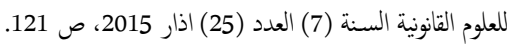

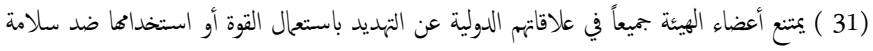

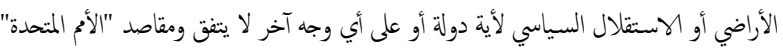

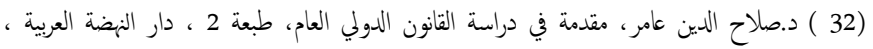
1995، ص 726 